\title{
Lidil
}

Revue de linguistique et de didactique des langues

$62 \mid 2020$

Recherches actuelles en didactique du lexique : avancées, réflexions, méthodes

\section{Mettre en œuvre des activités de classification de verbes de déplacement à l'école primaire}

Implementing Activities of Classification of Motion Verbs in Primary School

Michel Aurnague et Claudine Garcia-Debanc

\section{(2) OpenEdition}

Journals

Édition électronique

URL : http://journals.openedition.org/lidil/8266

DOI : $10.4000 /$ lidil.8266

ISSN : 1960-6052

Éditeur

UGA Éditions/Université Grenoble Alpes

Édition imprimée

ISBN : 978-2-37747-226-0

ISSN : $1146-6480$

Référence électronique

Michel Aurnaque et Claudine Garcia-Debanc, « Mettre en œuvre des activités de classification de verbes de déplacement à l'école primaire », Lidil [En ligne], 62 | 2020, mis en ligne le 03 novembre 2020, consulté le 10 décembre 2020. URL : http://journals.openedition.org/lidil/8266 ; DOI : https:// doi.org/10.4000/lidil.8266

Ce document a été généré automatiquement le 10 décembre 2020.

(C) Lidil 


\title{
Mettre en œuvre des activités de classification de verbes de déplacement à l'école primaire
}

Implementing Activities of Classification of Motion Verbs in Primary School

\author{
Michel Aurnague et Claudine Garcia-Debanc
}

\section{Introduction}

1 Les résultats présentés dans cet article sont issus d'une recherche collaborative sur l'enseignement du lexique des verbes de déplacement dans le cadre du projet Didacdép («Didactique de la description du déplacement»). Le projet a débuté de 2011 à 2013 dans six classes de CE2 et de cycle 3 (élèves de 8 à 11 ans) de Toulouse et de sa banlieue. Il s'est poursuivi, en 2014 et 2015, par une première phase d'analyse des données sous un angle plus spécifiquement linguistique (Aurnague \& Garcia-Debanc, 2016a, 2016b). Nous nous sommes ensuite penchés sur les dimensions didactiques de cette recherche collaborative (effets de l'ingénierie linguistique sur les acquisitions des élèves, intervention enseignante, réemploi du lexique, etc.), dimensions dont rend compte en partie la présente contribution.

2 Le choix du lexique du déplacement tient, en premier lieu, au rôle que joue l'espace dans notre rapport au monde et aux nombreux marqueurs lexicaux et grammaticaux présents dans les langues, notamment en français, pour référer à ce domaine. Cette motivation praxéologique est confirmée par la diversité des écrits impliquant la description de déplacements auxquels sont confrontés les élèves durant leur scolarité à l'école primaire et au début du collège : récits fictionnels (Masseron, 2001), mais aussi descriptions ou explications dans différents domaines disciplinaires, notamment en biologie (Laparra, 2005).

3 La seconde raison de ce choix réside dans la nécessité, pour mettre en œuvre un enseignement lexical cohérent, de disposer d'un modèle suffisamment précis du 
domaine notionnel enseigné et de son organisation dans la langue. Ce modèle linguistique permet de contrôler le matériau proposé aux élèves pour l'étude du lexique, en langue mais aussi en discours, à travers le choix de listes de mots ou d'extraits d'œuvres littéraires comportant un matériau lexical pertinent ainsi que la mise au point des consignes qui suscitent les activités. Il facilite également l'analyse et l'interprétation des données collectées au cours du protocole.

Plus généralement, on attend du modèle linguistique qu'il fournisse aux enseignant.e.s et aux élèves des clés à partir desquelles ils pourront accéder à l'organisation fine des unités linguistiques au sein du lexique. Il s'agira, par exemple, de dépasser le registre des relations sémantiques généralement étudiées (synonymie, antonymie, hyperonymie, etc.) pour découvrir la relation lexicale de "troponymie » s'établissant entre verbes (Miller \& Fellbaum, 1992) ${ }^{1}$. On pourra, concomitamment, entrevoir la possibilité qu'un verbe appartienne à plusieurs regroupements sémantiques, définissant par là même des classes non exclusives d'unités lexicales.

Dans cette contribution, nous nous demandons dans quelle mesure des activités de classification de listes de verbes de déplacement et les échanges oraux auxquels donne lieu la justification de ces regroupements contribuent à aider les élèves à expliciter la signification et les contextes d'emploi de chacun de ces verbes de sens proche et à en préciser les conditions d'emploi. Nous analysons les interventions de l'enseignant.e qui guident ce travail d'explicitation du sens des unités lexicales et nous nous interrogeons sur les modèles sous-jacents pouvant les expliquer.

6 Après avoir présenté les principes et le modèle linguistique qui sous-tendent l'ingénierie didactique proposée (section 2), nous décrivons les interventions enseignantes au cours d'une tâche de classification et nous en analysons les effets (section 3). Nous concluons en nous interrogeant sur les connaissances et compétences requises chez les enseignant.e.s pour mettre en œuvre un enseignement rénové du lexique (section 4).

\section{Une ingénierie didactique prenant appui sur un modèle linguistique robuste}

\subsection{Quelques principes généraux pour l'enseignement du lexique}

Les recherches didactiques sur l'enseignement du lexique des trente dernières années (pour une recension, on pourra consulter Garcia-Debanc, Masseron \& Ronveaux, 2013 ; Grossmann, 2011 et Nonnon, 2012) ont formulé un certain nombre de principes qui font l'objet d'un consensus et qui sous-tendent les activités d'enseignement proposées ici dans l'ingénierie didactique construite dans le cadre de la recherche Didacdép. Elles préconisent de ne pas isoler le lexique des autres composantes de la langue (syntaxe, énonciation et discours) et d'intéresser les élèves au choix des unités lexicales dans le processus même de production langagière, en écriture notamment (Garcia-Debanc, Duvignau, Dutrait \& Gangneux, 2009 ; Garcia-Debanc \& Chourau, 2010 ; Garcia-Debanc \& Gangneux, 2015). Les objets d'étude sont, de ce fait, non les mots isolés, mais des unités lexicales dans leurs propriétés combinatoires (Cusin-Berche, 1999). Les activités de contextualisation, décontextualisation, recontextualisation ainsi que de paraphrase occupent une place centrale dans l'enseignement du lexique. 
8 L'enseignement du lexique a un caractère systématique et explicite et, par conséquent, fait l'objet de séances spécifiques permettant de travailler sur les unités lexicales, (a) d'une part, en discours, par l'observation d'occurrences dans des productions orales d'expert.e.s ou d'élèves et des textes littéraires, relevant de la littérature de jeunesse ou de la littérature patrimoniale, ou des écrits documentaires en relation avec les différentes disciplines scolaires, (b) d'autre part, en langue, avec l'appui de différents dictionnaires, papier ou électroniques, destinés aux enfants ou aux adultes.

9 Si l'on tire les leçons des expérimentations conduites par des psycholinguistes, notamment celles de Lieury (1997) sur les conditions de mémorisation à long terme de nouvelles unités lexicales par des publics scolaires de bas niveau de qualification dans l'enseignement professionnel, sont acquises de façon durable les unités lexicales rencontrées en réception à l'oral et à l'écrit et employées à l'oral et à l'écrit. Elles sont en réseau avec le vocabulaire antérieurement maitrisé, ce qui nécessite, pour l'enseignant.e, de mettre en place des situations lui permettant de connaitre le vocabulaire déjà là chez chacun.e de ses élèves, qu'il soit actif, c'est-à-dire employé, ou passif, c'est-à-dire compris. L'acquisition à long terme nécessite aussi une réactivation permanente du vocabulaire déjà appris. De ce fait, l'enseignement lexical proposé ici repose à la fois sur des activités d'enseignement en voie longue, avec l'observation de corpus d'occurrences choisis par l'enseignant.e donnant lieu à des classifications dont les critères sont explicités par les élèves, et sur des activités ritualisées, brèves mais répétées, qui permettent le réemploi des unités lexicales dont les élèves ont antérieurement observé les propriétés sémantiques, morphologiques ou syntaxiques.

10 L'hypothèse est faite que le regroupement d'un ensemble de verbes au cours d'activités de classification oblige les élèves à la recherche de traits sémantiques communs. Les discussions auxquelles donnent lieu les différences de regroupements d'un élève à l'autre ou d'un groupe d'élèves à l'autre conduisent à une exploration des conditions d'emploi de chacun de ces verbes. Ces réemplois répétés dans des contextes nombreux participent à l'acquisition des unités lexicales. Les rectifications éventuelles des constructions verbales erronées permettent également de préciser les conditions d'emploi.

11 Du point de vue de la programmation des activités lexicales, l'organisation du système de la langue en différents niveaux d'analyse, telle que l'ont mise en évidence les travaux linguistiques, invite à prendre en compte ces différents niveaux d'analyse : la sémantique et la morphologie - celle-ci ayant souvent des incidences orthographiques -, mais aussi la syntaxe, l'étymologie et l'histoire des mots ainsi que la lexicographie. Les recherches didactiques (Garcia-Debanc, Masseron \& Ronveaux, 2013; Grossmann, 2011; Nonnon, 2012) convergent pour indiquer que les unités lexicales travaillées doivent être choisies en lien avec leur fréquence d'emploi ou leur polysémie: enseigner le lexique n'est pas enseigner des mots rares. Il importe également de veiller à la diversité des catégories grammaticales des unités lexicales étudiées, de façon à ce que l'enseignement du lexique ne privilégie pas les noms par rapport aux adjectifs, aux connecteurs ou aux verbes, ces derniers présentant un intérêt syntaxique particulier, à savoir l'analyse de leurs diverses constructions syntaxiques possibles.

12 Ces différents principes sont mis en acte dans l'ingénierie didactique que nous avons conçue et que nous présenterons infra en 2.3. Elle prend appui sur un modèle linguistique robuste. 


\subsection{Un modèle linguistique pour constituer des corpus d'observation}

13 Le domaine retenu pour cette recherche collaborative est, comme indiqué plus haut, celui de la description du déplacement et, plus exactement encore, du déplacement " autonome " d'une entité mobile. Il doit être distingué du déplacement "causé » exprimé par des verbes tels que amener, apporter, conduire, emporter, trainer ou transporter. D'un point de vue syntaxique, l'expérimentation porte, pour l'essentiel, sur des verbes intransitifs, transitifs «indirects » inclus : aller (+ Prép), arriver, déraper, descendre, entrer, foncer, se hisser, partir, ramper, se rendre, sortir, etc.

$\mathrm{Au}$ cours des dernières décennies, les recherches en syntaxe et sémantique sur l'expression du déplacement ont régulièrement mis en regard les verbes de déplacement au sens strict (arriver, partir, sortir, se rendre) et ceux qui dénotent la manière de se déplacer (foncer, grimper, marcher, zigzaguer), dans le sillage sans doute de l'opposition fondatrice, dans la description de l'espace dynamique (Talmy, 1985), entre référence au trajet ("path» en anglais) vs référence à la manière. Le cadre théorique adopté ici pour circonscrire les verbes et procès de déplacement au sens strict fait appel aux notions de changement de relation locative élémentaire (Boons, 1987) et de changement d'emplacement (Aurnague, 2008). Une relation locative élémentaire est exprimée par une préposition ou locution prépositionnelle spatiale du français, l'évaluation d'un possible changement de relation locative étant effectuée par rapport à l'entité-site ou entité localisatrice du procès décrit (ex. : Max est entré dans le parc). Un changement d'emplacement intervient dès lors que le verbe utilisé implique une modification de la position de l'entité-cible ou entité localisée dans le cadre de référence terrestre (ex. : Max a marché quelques mètres dans le parc). La combinaison de ces notions permet de définir quatre catégories de prédicats de mouvement/ déplacement. La recherche collaborative a porté sur les deux catégories les plus étroitement liées à la dynamicité spatiale et qui sont, en conséquence, les plus représentatives de la notion de déplacement: celle des changements de relation et d'emplacement (déplacement strict) et celle des changements d'emplacement, incluant de nombreux verbes de manière de déplacement, de même que des verbes directionnels. Deux listes de verbes ont été constituées sur ces bases :

- aller (à), s'en aller, aboutir, s'échapper, accéder, s'enfuir, arriver, entrer, partir, parvenir, pénétrer, se rendre, sortir, venir, atteindre, fuir, quitter (déplacement strict, liste A) ;

- avancer, couler, courir, dégringoler, déraper, descendre, foncer, dévaler, glisser, grimper, ramper, se hisser, reculer, rouler, monter, se trainer, marcher, nager, patiner, skier (changement d'emplacement, liste B).

La liste A comprend quatorze verbes intransitifs (de fait, transitifs indirects) de changement de relation et d'emplacement, deux verbes transitifs directs (atteindre et quitter) et un verbe entrant dans des constructions intransitives aussi bien que transitives directes (fuir). Elle associe des items d'usage fréquent (ex. : aller (à), arriver, partir, sortir, venir) à différentes unités lexicales moins fréquentes (ex. : aboutir, accéder, se rendre). Sur un plan sémantique, cette liste se partage entre prédicats dénotant des changements de relation de " polarité » initiale (ex. : s'en aller, s'échapper, partir, sortir) et prédicats dénotant des déplacements de polarité finale (ex. : aller (à), arriver, entrer, venir) (Aurnague, 2008, 2012). 
Compte tenu de la richesse et de la diversité des verbes de changement d'emplacement, la liste $\mathrm{B}$ a, de son côté, été élaborée à partir des quatre traits constitutifs de la propriété de " tendancialité » (Aurnague, 2008, 2012) : vitesse (courir), opposition à une force ou difficulté (ramper), direction ou déplacement linéaire orienté (reculer) et entrainement par une force (glisser) ${ }^{2}$. Un même verbe peut combiner plusieurs de ces traits dans son contenu sémantique (dégringoler : vitesse, direction, entrainement par une force). Seize des vingt verbes de la liste B présentent la propriété de tendancialité, alors que les autres verbes (marcher, nager, patiner, skier) font appel à d'autres éléments de sens, tels que le médium/milieu dans lequel l'entité-cible évolue, l'instrument ou la «partomotion» (mouvement relatif des parties de la cible au cours du déplacement). Les verbes de la liste B sont majoritairement intransitifs et uniquement six des vingt items sont susceptibles de donner lieu à des constructions intransitives ou transitives (dégringoler, descendre, dévaler, grimper, monter et, éventuellement, skier). Les unités lexicales retenues comprennent des verbes courants (ex. : avancer, couler, foncer, glisser, monter) ainsi que des verbes d'usage moins fréquent (dévaler, se hisser).

\subsection{Présentation des différentes tâches de l'ingénierie}

17 Les différentes tâches de l'ingénierie didactique, au nombre de sept, ont fait l'objet d'une présentation détaillée dans d'autres publications (Aurnague \& Garcia-Debanc, 2016a, 2016b; Garcia-Debanc \& Aurnague, 2020). Nous nous bornerons à contextualiser les séances de classification des verbes de déplacement de la liste B qui font ici l'objet des analyses.

Après deux tâches de production écrite, respectivement de phrases et d'un texte, permettant d'établir une évaluation diagnostique (tâches 1 et 2) et une observation des emplois des verbes de déplacement en discours, dans un album de jeunesse ou un texte patrimonial (tâche 3), sont proposées deux tâches de classification sémantique de verbes de déplacement, respectivement avec les verbes de la liste A (tâche 4) puis ceux de la liste B (tâche 5). Ces classifications sémantiques, tâches sémasiologiques, qui vont du signe linguistique à la signification, sont prolongées par une tâche onomasiologique, sous la forme d'activités ritualisées pour un réemploi dans des phrases inventées de verbes de la liste B (tâche 6). La séquence se clôt par une nouvelle tâche de production écrite de texte, permettant d'évaluer le réemploi des verbes de déplacement travaillés dans la séquence.

Cette ingénierie didactique a été mise en œuvre dans six classes de CE2 et de cycle 3. Les enseignant.e.s de ces classes ont une expérience professionnelle de durée variable ${ }^{3}$.

Les productions des élèves en réponse aux tâches de classification des listes de verbes $A$ et $\mathrm{B}$ et du rituel « Léon » (liste $\mathrm{B}$ ) ont, pour une partie d'entre elles, déjà donné lieu à une étude d'un point de vue essentiellement linguistique (Aurnague \& Garcia-Debanc, 2016a, 2016b). L'examen des résultats se focalise ici sur la dimension didactique. 


\section{Analyse des interventions de trois enseignant.e.s dans une tâche de classification : des conceptions différentes de la langue}

21 Les transcriptions des échanges captés dans une classe de CE2 (désormais «classe 1 ») et deux classes de CM1-CM2 (désormais «classe 2 » et "classe 3 »), au cours de trois séances de classification des verbes de la liste $\mathrm{B}$, ont fait l'objet d'une analyse détaillée. La multiplicité des traits sémantiques potentiellement repérables pour les verbes de cette liste $\mathrm{B}$, contrairement à ceux de la liste $\mathrm{A}$, qui comportent tous des caractéristiques relevant de la polarité, autorise un foisonnement de classifications possibles et la nécessité d'une méthode pour gérer la mise en commun de classifications très diverses. Nous considèrerons successivement la contextualisation du travail de classification dans les trois classes (3.1), le nombre et la diversité des classifications proposées par les différents groupes d'élèves (3.2), en mettant en relation celles-ci avec la nature des interventions des enseignantes (3.3). Ces analyses nous permettront d'inférer les représentations que se font les deux enseignantes des classes de cycle 3 de la tâche de classification et, plus largement, du système de la langue (3.4).

\subsection{Contextualisation du travail de classification dans les trois classes}

Les verbes de la liste B ont été présentés aux élèves selon l'ordre alphabétique des items.

Les séances débutent par une mise en contexte au cours de laquelle sont remobilisées les connaissances antérieurement travaillées. Les trois enseignantes interagissent avec les élèves afin de réactiver le contenu du travail déjà réalisé sur les verbes de déplacement au cours des séances précédentes et des classifications lexicales auxquelles il a donné lieu. Cette réactivation a été un peu différente selon les classes :

- dans les classes 1 et 3 : inventaire des verbes de déplacement utilisés dans les textes narratifs individuels rédigés par les élèves en réponse à la tâche 2 ; en complément, dans la classe 1 , recherche d'autres verbes de déplacement dans des dictionnaires et rappel des critères de classification des verbes de la liste A ;

- dans la classe 2 : observation des emplois de verbes de déplacement dans l'album L'épave du Zéphyr de Chris Van Allsburg, puis rappel de la classification de ces verbes.

Dans les trois classes, une grande partie des questions et discussions que posent les élèves dans la phase de présentation des consignes porte sur la nature des regroupements à réaliser. Taille attendue des regroupements? Classifications fondées sur des propriétés sémantiques plutôt que morphosyntaxiques? Proposition d'entêtes susceptibles de mettre en évidence l'unité de sens de chaque catégorie créée?

La classification est d'abord réalisée individuellement dans la classe 1. Dans la classe 3, les élèves interagissant oralement pendant qu'ils procèdent à des classifications individuelles, tandis que, dans la classe 2 , ils procèdent à une classification en groupes.

La comparaison de ces trois séances fait apparaitre d'importants écarts de conception de l'organisation du lexique chez les enseignantes menant ces séances, écarts qui se manifestent par des interventions différentes auprès des élèves et aboutissent à des 
classifications dont la structure et le contenu varient quantitativement selon les classes, comme en attestent les données d'ensemble présentées en 3.2.

\subsection{Des différences entre les classifications proposées par les différents groupes d'élèves dans les trois classes}

Étant donné la multiplicité de traits sémantiques des verbes de la liste B et la possibilité, pour nombre d'entre eux, d'appartenir à plus d'une catégorie, nous avons fixé notre attention sur les deux propriétés spécifiques suivantes :

- le nombre de catégories de verbes par classification produite en groupe ;

- le nombre de propriétés ou familles de propriétés sémantiques mobilisées par chaque classification.

Ces critères sont, au moins en partie, indépendants. En effet, une classification faisant apparaitre un nombre important de catégories (ex. avec 6 catégories : se déplacer vers le haut; vers le bas; horizontalement; sur le sol; dans l'eau ; dans les airs) peut baser les distinctions qu'elle opère sur un ensemble limité de propriétés de sens (propriétés de direction et de médium/milieu dans cet exemple).

Les deux critères ainsi délimités ont été appliqués à l'ensemble des classifications proposées. Le tableau 1 ci-dessous recense, pour chacune des trois classes, les classifications proposées par les groupes d'élèves en fonction du nombre de catégories qu'elles comportent (premier critère).

Tableau 1. - Répartition des classifications par nombre de catégories créées.

\begin{tabular}{|l|l|l|l|}
\hline Nombre de catégories & $1-2$ & $3-5$ & $6-9$ \\
\hline Classe 1 - nombre de classifications & & $6(100 \%)$ & \\
\hline Classe 2 - nombre de classifications & & $4(57,14 \%)$ & $3(42,86 \%)$ \\
\hline Classe 3 - nombre de classifications & $1(14,28 \%)$ & $3(42,86 \%)$ & $3(42,86 \%)$ \\
\hline
\end{tabular}

Le tableau 2 ci-dessous comptabilise, pour sa part, les mêmes classifications selon le nombre de propriétés ou familles de propriétés sémantiques sous-tendant les catégories dégagées dans chaque classification (second critère). L'identification des éléments de sens plus élémentaires qui structurent les classifications est réalisée grâce au modèle linguistique présenté en section 2.2 et aux traits sémantiques pertinents que celui-ci a permis de recenser.

Tableau 2. - Répartition des classifications par nombre de propriétés sémantiques mobilisées.

\begin{tabular}{|l|l|l|l|l|l|}
\hline Nombre de propriétés & 1 & 2 & 3 & 4 & 5 \\
\hline Classe 1 - nombre de classifications & $2(33,33 \%)$ & $4(66,66 \%)$ & & & \\
\hline Classe 2 - nombre de classifications & $1(14,28 \%)$ & $3(42,86 \%)$ & $2(28,57 \%)$ & & $1(14,28 \%)$ \\
\hline
\end{tabular}




\begin{tabular}{|l|l|l|l|l|l|}
\hline Classe 3- nombre de classifications & $1(14,28 \%)$ & & $2(28,57 \%)$ & $3(42,86 \%)$ & $1(14,28 \%)$ \\
\hline
\end{tabular}

Une observation globale de ces données fait apparaitre une différence importante entre la classe 1 (CE2) et les classes 2 et 3 (CM1-CM2) pour ce qui est du nombre de catégories ou regroupements créés par classification (tableau 1) et une augmentation continue, d'un niveau scolaire à l'autre, de l'éventail de propriétés sémantiques activées (au-delà de 2 puis au-delà de 3 propriétés : tableau 2). Ces différences peuvent être imputées au niveau scolaire, mais aussi, comme nous le montrons en 3.3, à l'intervention enseignante, qui semble bien agir sur la structure et la nature des classifications élaborées en groupes par les élèves, telles que mises en lumière par les données cidessus. Deux visions bien distinctes de la classification paraissent, en effet, guider l'intervention enseignante : application au lexique de schémas et contraintes externes à la langue $v s$ prise en compte de la complexité intrinsèque de l'organisation lexicale.

\subsection{Nature et effets des interventions des enseignantes au cours de l'activité dans les trois classes}

Dans la classe 1, les 6 classifications comprennent 3 à 5 catégories de verbes (tableau 1). Les propriétés de sens ou familles de propriétés organisant ces catégories sont au nombre de 4 : direction (ex. d'entêtes: avancer/ $\rightarrow$, monter), médium/milieu (ex. d'entêtes : sur l'eau, sur la terre), vitesse (ex. d'entêtes : vite, lentement) et partomotion/ glisse (mouvement relatif des parties de la cible dans le cadre d'un déplacement mettant en jeu le glissement sur une surface; ex. d'entête : glisser). Les propriétés de directionnalité et de médium/milieu déjà connues des élèves à travers les activités précédentes sous-tendent, à elles seules, $77 \%$ des regroupements de verbes élaborés. De plus, la combinaison de plusieurs propriétés de sens au sein d'une même classification ne dépasse pas 2 propriétés (tableau 2).

L'enseignante 1 semble relativement peu intervenir durant la classification par groupes. Les suggestions qu'elle fait aux élèves font plusieurs fois appel à la notion de direction via le recours à des flèches. Elle associe le critère de "direction ", qu'elle suggère, à celui de polarité des verbes de déplacement, ce qui n'est pas tout à fait exact (voir section 2.2).

Dans la phase de mise en commun, au cours de la présentation des classifications par les rapporteurs des divers groupes, elle procède à un enregistrement des critères proposés sous forme d'inventaire, sans critiques ou suggestions nouvelles permettant à d'autres traits d'émerger. Plus généralement, les critères classificatoires suggérés reprennent les propriétés ayant émergé pendant les activités précédentes de la séquence d'enseignement : direction et médium/milieu.

Les classifications proposées par les élèves de la classe 1 semblent donc refléter les propriétés sémantiques privilégiées par l'enseignante, à savoir directionnalité et médium/milieu. Cette séance parait s'inscrire dans la simple continuité d'activités antérieures ayant mobilisé ces deux propriétés sémantiques. Le chercheur ne peut pas en inférer une vision spécifique de l'organisation lexicale chez l'enseignante.

Contrairement aux classifications de la classe 1, 3 des 7 classifications élaborées en groupes dans la classe $2(42,86 \%)$ font apparaitre plus de 5 catégories de verbes (tableau 1). Deux nouvelles propriétés sémantiques (ou familles de propriétés) viennent 
s'ajouter à celles utilisées dans la classe 1: la force ou dynamique des forces (ex. d'entêtes : un effort (opposition à une force/difficulté); accident, tomber (entrainement par une force)) et le trait essentiellement contextuel de déplacements liés au sport (ex. d'entête : sport). Les propriétés de directionnalité et de médium/milieu mises en œuvre dans la classification des verbes de l'album L'épave du Zéphyr dans des séances antérieures demeurent encore, et de loin, les plus mobilisées (près de $69 \%$ des regroupements). Malgré la variété des propriétés ou familles de propriétés disponibles ( 6 au total), il est frappant de constater que seules 3 classifications sur 7 mobilisent plus de 2 propriétés de sens $(42,85 \%$; voir tableau 2$)$, une seule classification $(14,28 \%)$ recourant à plus de 3 propriétés.

L'enseignante 2 intervient beaucoup au cours de la classification par groupes ainsi que lors de la mise en commun - où c'est elle qui présente les affiches des différents groupes -, et ce en deux directions partiellement contradictoires. D'une part, elle encourage les élèves à faire émerger de nouvelles propriétés sémantiques - pas encore travaillées - permettant de regrouper certains verbes, comme dans le cas de l'opposition à une force ou difficulté (voir section 2.2 et plus bas). D'autre part, elle tient à ce que les classifications produites s'appuient, tout au plus, sur deux "dimensions " sémantiques distinctes, à partir desquelles l'ensemble des verbes de la liste B devrait pouvoir être organisé. Elle qualifie de "problématiques" les classifications ayant recours à plus de deux familles de propriétés ou à des propriétés jugées non compatibles : "[...] mais le classement il pose des problèmes comme ça »; " [...] mais c'est un peu tout mélangé et on ne sait pas très bien comment l'organiser ». Elle invite explicitement les élèves à classer les verbes à partir d'une seule famille de propriétés (p. ex. la direction : « vers le haut », « vers le bas », « horizontalement ») ou au moyen de tableaux à double entrée (p. ex. la direction [voir ci-dessus] et le médium/ milieu [« sur le sol », « dans l'eau »]). Elle suscite donc une utilisation systématique d'un nombre limité de critères de classification et des catégorisations exclusives.

Dans la classe 3, si la répartition des classifications en fonction du nombre de catégories de verbes créées est sensiblement la même que dans la classe 2 (tableau 1), le poids des classifications utilisant plus de $2(85,71 \%)$, voire plus de 3 familles de propriétés de sens (57,14\%), est nettement plus élevé que dans les autres classes (tableau 2). Autrement dit, non seulement les élèves construisent des classifications comprenant un nombre important de catégories (comme dans la classe 2), mais ils combinent aussi plus volontiers les traits sémantiques à l'intérieur des classifications (contrairement à la classe 2). L'influence des critères de médium/milieu et de direction reste encore forte (61,53\% des regroupements), tout en étant moins importante que dans les deux autres classes. La propriété sémantique de mode ou moyen de transport - nettement contextuelle ici (ex. d'entêtes : transport, en voiture!) - s'ajoute à celles déjà présentes dans les productions de la classe 2 .

L'enseignante 3 intervient, comme l'enseignante 2, de façon récurrente dans la séance : son rôle consiste, pour l'essentiel, à s'assurer que les élèves opèrent des regroupements de verbes et dégagent des propriétés sémantiques censées justifier ces regroupements, qui sont ensuite listées. Elle laisse les élèves libres dans leurs choix («Mettez-vous d'accord entre vous. Il faut mettre un titre à ceux que vous avez mis ensemble »; «C'est entre vous qu'il faut décider. Moi, je ne vais pas vous dire quelle est la bonne solution, il n'y en a pas ») et ne porte pas de jugement sur la nature et le nombre de familles de propriétés activées dans une même classification. 


\subsection{Deux conceptions différentes de la classification des verbes chez les enseignantes des classes 2 et 3}

40 Les interventions des deux enseignantes de cycle 3 dessinent ainsi des visions opposées de la classification linguistique, qui, comme on vient de le voir, ont des répercussions mesurables sur les productions des élèves. Dans la classe 2, la classification des items lexicaux est supposée répondre à des schémas prédéfinis imposant des contraintes sur le nombre et la compatibilité des propriétés de sens susceptibles de structurer la liste de verbes. Dans la classe 3, la complexité interne des items de la liste B est entièrement assumée, avec, en particulier, la possibilité que ceux-ci puissent s'organiser selon une variété de dimensions sémantiques.

41 Les discussions qui accompagnent et concluent la mise en commun des classifications sont, à ce titre, révélatrices. L'enseignante 2 insiste à plusieurs reprises sur la complexité et la difficulté de la tâche («[...] c'était pas facile, ni si évident que ça »; « C'est très compliqué [...]»), alors que l'enseignante 3 termine la séance en montrant aux élèves que les items lexicaux regroupés dans une catégorie sur la base d'une même propriété de sens diffèrent souvent par d'autres aspects de leur contenu sémantique (Aurnague \& Garcia-Debanc, 2016b), soulignant ainsi, indirectement, la variété des critères possibles de regroupement et l'appartenance éventuelle d'un item à plusieurs catégories en fonction du critère choisi. Pour ce faire, l'enseignante 3, au cours de la mise en commun, se focalise sur les verbes ramper, glisser et se trainer rassemblés sous l'entête au sol et suscite un échange avec les élèves en posant la question suivante: «[...] Donc, ils nous disent tous qu'on va se déplacer au sol. On est tous d'accord. Mais est-ce qu'ils veulent dire, tous, exactement la même chose ? Pourquoi est-ce qu'alors, on utilise trois mots différents ? [...]. » Elle sensibilise ainsi ses élèves à la spécificité des significations de mots de sens proches et aux conditions précises de leur emploi. On peut raisonnablement penser que de telles interventions développent aussi la « sensibilité lexicale » des élèves (Tremblay \& Gagné, 2019).

\section{Conclusion : quelles connaissances et compétences sont requises chez les enseignant.e.s pour une mise en œuvre d'un enseignement rénové du lexique?}

L'enseignement du lexique reste un parent pauvre parmi les activités d'étude de la langue. Alors que l'orthographe, la syntaxe et la morphologie flexionnelle font le plus souvent l'objet d'un enseignement structuré, avec une programmation explicite et des activités fréquentes et ritualisées, l'enseignement du lexique est souvent moins systématique (Grossmann, 2011). Il prend fréquemment la forme d'exercices prélevés dans des manuels. L'ingénierie didactique que nous avons proposée ici se distingue d'une telle pratique par de nombreux traits: recensement du vocabulaire actif disponible avant la mise en place de la séquence, observations d'emplois en langue et en discours, notamment en littérature de jeunesse, activités de classification d'unités lexicales sélectionnées selon des critères de fréquence et de présence de traits sémantiques particuliers et échanges oraux sur ces classifications, élaboration d'aides lexicales pouvant servir d'appui dans les tâches de production écrite (Garcia-Debanc, 
2013), activités de réemploi nombreuses des unités lexicales travaillées, à l'oral et à l'écrit, évaluation du lexique acquis au cours de la séquence.

Nous avons vu combien les incitations des enseignant.e.s au cours de la réalisation en groupes d'une affiche présentant les regroupements de verbes et de la discussion autour de ces affiches dans la phase de mise en commun ont un effet important sur la nature des classifications réalisées par les élèves. Nous avons montré également l'influence de la conception que se fait l'enseignant.e de l'activité de classification elle-même et du système de la langue. On peut se demander quelles connaissances et compétences professionnelles sont nécessaires à l'enseignant.e pour mettre en œuvre et concevoir un enseignement de ce type. On peut raisonnablement penser que sont requises à la fois des connaissances linguistiques, des compétences professionnelles et des attitudes de curiosité par rapport au fonctionnement de la langue.

Du point de vue des connaissances linguistiques, l'enseignement du lexique requiert la maitrise par l'enseignant.e de l'architecture du lexique en langue et des diverses dimensions de son étude (morphologie, sémantique, syntaxe, énonciation, étymologie, lexicographie, etc.), dans leur autonomie et leur solidarité. Cette connaissance permet de caractériser l'approche choisie et de concevoir une programmation d'ensemble cohérente. Il est requis aussi de maitriser les paramètres qui permettent de procéder à une classification des unités lexicales, ici les notions de changement d'emplacement et de changement de relation locative élémentaire, de polarité initiale ou finale ainsi que d'autres traits sémantiques de classification. Les connaissances linguistiques permettent de sélectionner les corpus soumis à l'observation des élèves, extraits de discours, notamment littéraires ou listes de mots en langue, de comprendre et d'interpréter les intuitions linguistiques des élèves et leurs erreurs lexicales, de susciter des discussions entre eux, de les aider à formaliser des régularités en système et enfin, d'organiser un entrainement favorisant un réemploi des unités lexicales travaillées dans diverses situations, à l'oral et à l'écrit.

Un des principaux obstacles rencontrés dans la mise en œuvre d'une séance d'enseignement du lexique tient à la nature des intuitions sémantiques des élèves et à la confusion courante qu'ils font entre le mot et son référent ou, ici, entre le sémantisme d'un verbe et les éléments de sens apportés par le cotexte ou le contexte situationnel. Parfois, une grande partie, voire l'ensemble d'un regroupement lexical peut être motivé par des paramètres contextuels. Pour surmonter cet obstacle récurrent, l'enseignant.e doit amener à distinguer la signification d'une unité lexicale en langue de sa signification dans un contexte particulier. La décontextualisation vise à démontrer qu'une propriété attribuée à un prédicat découle, en réalité, du contexte d'emploi spécifique imaginé par le ou les élèves. L'appui sur les collocations et l'axe syntagmatique constitue un moyen efficace d'aider les élèves à faire le départ entre sens lexical et emploi contextuel. L'analyse des extraits filmés des trois séances de classification de la liste B a mis en évidence l'importance de telles interventions en réponse aux propositions des élèves. Ainsi une élève de la classe 3 justifie-t-elle le rapprochement entre le verbe reculer et des prédicats dénotant la vitesse (foncer, courir) en disant que « reculer, si on fait une course pour reculer, on va vite!». L'enseignante de la classe 3 répond à ce commentaire par la question suivante : «Alors, je recule à toute vitesse par exemple. Est-ce que c'est le verbe qui te montre que tu vas vite ? [...]. » Les opérations de contextualisation $v s$ décontextualisation semblent centrales pour l'accès maitrisé à la construction du sens: elles interviennent tout au long de la séquence 
d'enseignement, aussi bien au cours des activités de classification en langue que de l'observation des emplois en discours. Elles requièrent chez l'enseignant.e des compétences à distinguer signification de l'unité lexicale et emplois contextuels et, plus largement, forme linguistique, référence et signification.

Pour conduire un enseignement rénové du lexique, l'enseignant.e doit aussi manifester une attitude de curiosité par rapport au fonctionnement de la langue, une attention aux choix lexicaux, voire une certaine gourmandise par rapport aux mots, ce qu'Ophélie Tremblay propose d'appeler la «sensibilité lexicale » (Tremblay \& Gagné, 2019). La pratique régulière de lectures et de mémorisations de textes poétiques et de textes littéraires denses développe cette attention aux mots qui peut ainsi être transmise aux élèves.

L'enseignement du lexique est une préoccupation permanente et suscite des apprentissages incidents au gré des rencontres de mots nouveaux à la signification inconnue ou de mots polysémiques dans de nouvelles acceptions, mais il nécessite aussi un enseignement explicite, systématique et programmé sur des notions nodales. Le travail sur les verbes de déplacement présenté ici permet aux élèves de structurer et d'acquérir un lexique diversifié dans un domaine notionnel cognitivement fondamental, l'espace. En plus du vocabulaire ainsi acquis, dont l'amplitude est difficile à apprécier, la séquence didactique proposée présente aussi un intérêt méthodologique important, en amenant les élèves à inventer des contextes d'emploi des unités lexicales travaillées, à explorer leurs intuitions linguistiques sur les constructions syntaxiques et les conditions d'emploi d'un certain nombre de verbes plus ou moins fréquents et à réorganiser leur lexique mental pour y intégrer de nouvelles unités lexicales.

La méthodologie de construction de l'ingénierie didactique présentée ici peut être transférée à d'autres contenus lexicaux : un travail analogue peut être conduit sur les verbes indiquant l'acte de manger (Duvignau \& Garcia-Debanc, 2008), les verbes de parole (Charolles, 1976 ; Lamiroy \& Charolles, 2008) ou les verbes du lexique verbal des sentiments (Grossmann, Boch \& Cavalla, 2008 ; Masseron, 2008). De telles activités visent à favoriser l'emploi de verbes spécifiques, dont l'acquisition est plus tardive en langue 1 et en langue 2 que celle des verbes de base (Kihlstedt, 2008 ; Noyau, 2005). Mais au-delà de ces acquisitions lexicales spécifiques, une telle démarche permet aux élèves de verbaliser leurs intuitions sémantiques, de vérifier les conditions d'emploi et les constructions syntaxiques de ces verbes et, plus largement, de comprendre que le lexique est structuré en réseaux sémantiques et que le choix d'une unité lexicale s'opère parmi des unités lexicales de sens proches, chacune d'entre elles suscitant des effets de sens différents. Elle contribue ainsi à développer la curiosité, voire la gourmandise, dans le choix des unités lexicales, à l'oral et à l'écrit, et d'en apprécier toutes les nuances de sens. 


\section{BIBLIOGRAPHIE}

AuRnAGUE, Michel. (2008). Qu'est-ce qu'un verbe de déplacement ? Critères spatiaux pour une classification des verbes de déplacement intransitifs du français. Dans J. Durand, B. Habert \& B. Laks (dir.), Actes du Congrès mondial de linguistique française, CMLF'08 (p. 1905-1917, cd-rom). Paris : ILF \& EDP Sciences. <http://dx.doi.org/10.1051/cmlf08041>.

AuRnaGue, Michel. (2012). De l'espace à l'aspect : les bases ontologiques des procès de déplacement. Corela, HS-12. <https://doi.org/10.4000/corela.2846>.

AuRnAGUE, Michel \& GARCIA-DEBANC, Claudine. (2016a). Enseignement du lexique à l'école primaire et modélisations linguistiques : exemples d'activités portant sur des verbes de déplacement strict. Pratiques, 169-170. <https://doi.org/10.4000/pratiques.2990>.

Aurnague, Michel \& Garcia-Debanc, Claudine. (2016b). Les verbes de déplacement comme contenu d'enseignement du lexique à l'école primaire : modélisation linguistique et analyse de productions d'élèves. Dans F. Neveu, G. Bergounioux, M. H. Côté, J. M. Fournier, L. Hriba \& S. Prévost (dir.), Actes du $5^{e}$ Congrès mondial de linguistique française, CMLF 2016. Paris : ILF \& EDP Sciences. <http://dx.doi.org/10.1051/shsconf/20162707001>.

Boons, Jean-Paul. (1987). La notion sémantique de déplacement dans une classification syntaxique des verbes locatifs. Langue française, 76, 5-40.

Charolles, Michel. (1976). Exercices sur les verbes de communication. Pratiques, 9, 83-107.

CUSIN-BERCHE, Fabienne. (1999). La notion d'« unité lexicale » en linguistique et son usage en lexicologie. Linx, 40, 11-20.

DUVIGNAU, Karine \& GARCIA-DEBANC, Claudine. (2008). Un apprentissage du lexique verbal par proximité sémantique : quand la représentation lexicale facilite la tâche d'écriture. Dans F. Grossmann \& S. Plane (dir.), Les apprentissages lexicaux. Lexique et production verbale (p. 17-41). Villeneuve-d'Ascq : Presses universitaires du Septentrion.

GARCIA-DEBANC, Claudine. (2013). Les aides lexicales à la rédaction de textes : panorama historique et essai de typologie. Dans C. Garcia-Debanc, C. Masseron \& C. Ronveaux (dir.), Enseigner le lexique (p. 273-300). Namur : AIRDF \& Presses universitaires de Namur.

GARCIA-DEBANC, Claudine \& CHOURAU, Annie. (2010). Enseigner les verbes de déplacement pour l'écriture de récits : de l'analyse de besoins à la mise en place d'activités en classe de CM2. Recherches, 53, 139-158.

Garcia-Debanc, Claudine, Duvignau, Karine, Dutrait, Claire \& Gangneux, Michel. (2009).

Enseignement du lexique et production écrite : un travail sur les verbes de déplacement à la fin de l'école primaire. Pratiques, 141-142, 208-232.

GARCIA-DEBANC, Claudine \& GANGNEUX, Michel. (2015). L'enseignement de la synonymie à l'école primaire : état des lieux et recherches innovantes pour une articulation entre enseignement du lexique et production écrite. Études de linguistique appliquée, 178, 143-164.

Garcia-Debanc, Claudine, Masseron, Caroline \& Ronveaux, Christophe. (2013). L'enseignement du lexique : objets et pratiques. Dans C. Garcia-Debanc, C. Masseron \& C. Ronveaux (dir.), Enseigner le lexique (p. 15-32). Namur : AIRDF \& Presses universitaires de Namur. 
GARCIA-DEBANC, Claudine \& AuRnAGUE, Michel (2020). Quelle programmation des activités d'étude de la langue sur le lexique en fin d'école primaire pour susciter le réemploi en production écrite? Repères, 61.

GRossmanN, Francis. (2011). Didactique du lexique : état des lieux et nouvelles orientations.

Pratiques, 149-150, 163-183.

Grossmann, Francis, Boch, Françoise \& CAVALla, Cristelle (2008). Quand l'écriture n'empêche pas les sentiments... Quelques propositions pour mieux intégrer la dimension lexicale. Dans

F. Grossmann \& S. Plane (dir.), Les apprentissages lexicaux. Lexique et production verbale (p. 191-218). Villeneuve-d'Ascq : Presses universitaires du Septentrion.

KIHLSTEDT, Maria (2008). Stratégies compensatoires dans l'acquisition du lexique verbal en français. Dans F. Grossmann, M.-A. Paveau \& G. Petit (dir.), Didactique du lexique : langue, cognition, discours (p. 85-105). Grenoble : ELLUG.

LAMIROY, Béatrice \& CHAROLLES, Michel (2008). Les verbes de parole et la question de l'(in)transitivité. Discours, 2. <https://doi.org/10.4000/discours.3232>.

LAPARRA, Marceline. (2005). Capacités langagières en production non fictionnelle. Pratiques, 125-126, 139-156.

LIEURY, André. (1997). Mémoire et réussite scolaire. Paris : Dunod.

MASSERON, Caroline. (2001). Note critique sur les exercices de grammaire. Pratiques, 111-112, 209-236.

MASSERON, Caroline. (2008). Pour une topique de la peur : aspects psychologiques, sémiotiques, linguistiques. Dans F. Grossmann \& S. Plane (dir.), Les apprentissages lexicaux. Lexique et production verbale (p. 161-190). Villeneuve-d'Ascq : Presses universitaires du Septentrion.

Miller, George A. \& Fellbaum, Christiane. (1992). Semantic Networks of English. Dans B. Levin \& S. Pinker (dir.), Lexical and Conceptual Semantics (p. 197-229). Cambridge, MA / Oxford : Blackwell. NonNon, Élisabeth. (2012). La didactique du français et l'enseignement du vocabulaire, dans vingt ans de revues de didactique du français langue première. Repères, 46, 33-72.

NOYAU, Colette (2005). Le lexique verbal dans des restitutions orales de récits en français L2 : verbes de base, flexibilité sémantique, granularité. Dans F. Grossmann, M.-A. Paveau \& G. Petit (dir.), Didactique du lexique : langue, cognition, discours (p. 65-84). Grenoble : ELLUG.

TALMY, Leonard. (1985). Lexicalization Patterns: Semantic Structure in Lexical Forms. Dans T. Shopen (dir.), Language Typology and Syntactic Description: Grammatical Categories and the Lexicon (vol. 3, p. 57-143). Cambridge : Cambridge University Press.

TREMBLAY, Ophélie \& GAGNÉ, Andréanne (2019, août). Le concept de sensibilité lexicale en question : enjeux affectifs, théoriques et didactiques. Communication présentée au symposium « Concepts émergents, pratiques innovantes et réflexions méthodologiques actuelles en didactique du lexique » dans le cadre du $14^{\mathrm{e}}$ colloque de l'AIRDF, Lyon, France.

\section{NOTES}

1. La relation de troponymie - $\mathrm{du}$ grec tropos "manière, mode " - lie un lexème verbal superordonné (ex. : se déplacer) à un lexème sémantiquement apparenté quoique plus spécifique 
(ex. : se trainer). Cette notion peut ainsi prendre le relai, dans le domaine des verbes, de la relation d'hyperonymie.

2. La présence d'un ou plusieurs traits de tendancialité dans le sémantisme d'un verbe de changement d'emplacement conditionne la possibilité d'une interprétation (télique) incluant un changement de relation final lorsque le verbe est associé à un groupe prépositionnel locatif adéquat (Aurnague, 2008, 2012) : Max a couru/rampé/reculé/glissé sur la chaussée.

3. Quatre enseignantes maitres formatrices ont plus de dix années d'expérience professionnelle et attestent d'une certification de formatrices et deux enseignantes sont en première année d'enseignement.

\section{RÉSUMÉS}

Cette contribution traite des données collectées dans le cadre d'une recherche collaborative menée dans des classes de CE2 et de cycle 3 (élèves de 8 à 11 ans) de l'école primaire française. Faisant suite à des analyses réalisées sous un angle linguistique, elle se focalise sur le versant didactique des expérimentations en classe.

L'article débute par un rappel des principes ayant guidé l'ingénierie didactique avec, au premier plan, l'utilisation d'un modèle linguistique du domaine lexical objet de l'enseignement, celui des verbes de déplacement. Il se poursuit par une synthèse de l'analyse des extraits filmés des interventions de trois enseignant.e.s dans une activité de classification de verbes. Cette analyse met en lumière, de la part des enseignant.e.s, des visions différentes de l'organisation du lexique, qui ont une influence sur les classifications élaborées par les élèves.

L'article se conclut par une tentative de recensement des connaissances et compétences requises chez les enseignant.e.s, pour la mise en œuvre d'un enseignement rénové du lexique.

This contribution deals with the data collected within a collaborative research project conducted in French primary schools (three last years of primary school). Following several analyses made from a linguistic perspective, it focuses on the didactic aspect of the experimentations carried out in the classrooms.

The paper starts by recalling the principles that guided the didactic engineering with, at the forefront, the resort to a linguistic model of the lexical domain being taught, that of motion verbs. It follows with a synthesis of the analysis of film clips showing the interventions of three teachers in an activity of verb classification. This analysis brings to light different views of the semantic organization of the lexicon from the part of the teachers, these views having an impact on pupils' classifications.

The paper concludes with an attempt to identify the knowledge and skills required for teachers in order to be able to implement a renewed teaching of the lexicon.

\section{INDEX}

Keywords : vocabulary teaching, motion verbs, classification activities, vocabulary study in language, teachers' interventions

Mots-clés : enseignement du lexique, verbes de déplacement, activités de classification, étude du lexique en langue, intervention enseignante 


\section{AUTEURS}

\section{MICHEL AURNAGUE}

CLLE, Université de Toulouse, CNRS, UT2J, France

\section{CLAUDINE GARCIA-DEBANC}

CLLE, Université de Toulouse, CNRS, UT2J, France

SFR-AEF, INSPE Toulouse Occitanie Pyrénées, France 